Bull. Austral. Math. Soc.

VoL. 76 (2007) [243-262]

\title{
GENERALISED SYMMETRIES OF PARTIAL DIFFERENTIAL EQUATIONS VIA COMPLEX TRANSFORMATIONS
}

\author{
D. Catalano Ferraioli, G. Manno and F. Pugliese
}

We consider two systems of real analytic partial differential equations, related by a holomorphic contact map $H$. We study how the generalised symmetries of the first equation are mapped into those of the second one, and determine under which conditions on $H$ such a map is invertible. As an application of these results, an example of physical interest is discussed.

\section{INTRODUCTION}

It is a usual practice, when studying a given differential equation, to map it into another one via a contact map $[1,2,9,10]$. In fact, if one knows symmetries or conservation laws or explicit solutions of the transformed equation, one can recover the analogous objects for the first equation by reversing the transformation.

In some cases it is possible to map a real differential equation into another one by a complex contact transformation. For example, Laplace equation

$$
u_{x x}+u_{y y}=0
$$

is turned into the wave equation

$$
v_{\xi \eta}=0
$$

by the linear complex map

$$
\left\{\begin{array}{l}
\xi=x+i y \\
\eta=x-i y \\
v=u
\end{array}\right.
$$

or, rather, by its second order contact prolongation. Now, the general solution of (2) is

$$
v=f(\xi)+g(\eta)
$$

Received 31st January, 2007

We wish to thank A.M. Vinogradov for having proposed the problem. We also thank R. Alonso Blanco and F. Oliveri for stimulating discussions, and the Department of Mathematics of the University of Lecce for financial support. Finally we wish to thank warmly V. Rossi for his constant encouragement.

Copyright Clearance Centre, Inc. Serial-fee code: 0004-9727/07 \$A2.00+0.00. 
with $f, g$ arbitrary holomorphic functions of one complex variable. By reversing (3) one gets the following result: the general real analytic solution $u(x, y)$ of $(1)$ is of the form

$$
u=f(x+i y)+g(x-i y),
$$

with $f, g$ arbitrary holomorphic functions. Note that (4) is generically a complex valued function of real variables $x, y$. However, its real and imaginary part are real valued solutions of (1). The same holds when dealing with (classical or generalised) symmetries of the transformed equation. For example, the infinitesimal generalised symmetry $v_{\xi \xi} \frac{\partial}{\partial v}$ of (2) can be mapped, through the inverse map of (3), into the complex-valued operator $\left(u_{x x}-u_{y y}-2 i u_{x y}\right) / 4 \frac{\partial}{\partial u}$ whose real and imaginary part are symmetries of (1).

The above method for computing symmetries is frequently used in practice (see for instance various examples in [1]). However, such computations are of a formal nature: the aim of this paper is to present them in a rigorous geometrical framework, which we resume below.

Let

$$
\left\{F^{i}\left(x, u, u^{(1)}, \ldots, u^{(r)}\right)=0\right\}_{i=1, \ldots, s}
$$

be a $r$-th order system of partial differential equations in the unknown vector-valued function $u^{j}=u^{j}\left(x^{1}, \ldots, x^{n}\right), j=1, \ldots, m$ (in (5), with $u^{(k)}$ we denote the $k$-th order partial derivatives). We assume $F^{i}$ to be real analytic in some open domain $U$ of $\mathbb{R}^{N}$, $N=n+m\left(\begin{array}{c}n+r \\ r\end{array}\right)$. Under such assumption, $F^{i}$ can be extended to a holomorphic function $\widehat{F^{i}}: \widehat{U} \rightarrow \widehat{C}$, with $U \subset \widehat{U}$ and $\widehat{U}$ open set in $\mathbb{C}^{N}$ : such an extension is locally unique $([5,6])$. Let now $H_{0}: \mathbb{C}^{n+m} \rightarrow \mathbb{C}^{n+m}$ be a "fibrewise" biholomorphism, that is, one of the form:

$$
H_{0}: \begin{cases}\xi^{\lambda}=h^{\lambda}\left(x^{1}, \ldots, x^{n}\right), & \lambda=1, \ldots, n, \\ v^{j}=g^{j}\left(x^{1}, \ldots, x^{n} ; u^{1}, \ldots, u^{m}\right), & j=1, \ldots, m,\end{cases}
$$

with $h=\left(h^{1}, h^{2}, \ldots, h^{n}\right): \mathbb{C}^{n} \rightarrow \mathbb{C}^{n}$ being a biholomorphism. Then the contact prolongation $H: \mathbb{C}^{N} \rightarrow \mathbb{C}^{N}$ of $H_{0}$ maps the system $\left\{\widehat{F}^{i}=0\right\}_{i=1, \ldots, s}$ into

$$
\left\{\widehat{G^{i}}\left(\xi, v, v^{(1)}, \ldots, v^{(r)}\right)=0\right\}_{i=1, \ldots, s}
$$

with $\widehat{G^{i}}=\widehat{F^{i}} \circ H^{-1}$ being the holomorphic extensions of some real-analytic functions $G^{i}$ defined on (an open domain of) $\mathbb{R}^{N}$. Note that, under some dimensional assumptions (see section 2.2), functions $G^{i}$ are real-valued. Let now $X$ be a generalised symmetry of system

$$
\left\{G^{i}=0\right\}_{i=1, \ldots, \theta}
$$


and denote by $\widehat{X}$ its holomorphic extension. Then, as in the above example, the restrictions to $\mathbb{R}^{N}$ of the real and imaginary part of vector field $H^{-1}(\widehat{X})$ are symmetries of (5). Thus one gets a "multivalued" map from symmetries of (7) to those of (5). Obviously, such a map does not preserve linear independence. However, in section 2.2 we prove the main result of the paper (theorem 4), namely that, under some natural assumption on transformation (6), the map

$$
\left.X \longmapsto \operatorname{Re}\left(H^{-1}(\widehat{X})\right)\right|_{\mathbf{R}^{n}}+\left.\operatorname{Im}\left(H^{-1}(\widehat{X})\right)\right|_{\mathbf{R}^{n}}
$$

is invertible. This result allows the finding of wide subalgebras of real analytic symmetries of (5) starting from those of (7). An analogous result holds for recursion operators (theorem 6).

Some remarks are in order. First we note that, in general, neither the domain nor the range of (8) coincide with the whole algebras of symmetries. This is due to the fact that (the contact prolongation of) complex transformation (6) does not act on real symmetries of the transformed equation but, rather, on their holomorphic extensions. In other words, while $H$ is a pointwise correspondence between complexifications of (5) and (7), it induces a non-pointwise correspondence between the original real systems and their symmetries.

Secondly, recall that, from a geometrical point of view, a system of partial differential equations is a submanifold of a suitable jet bundle $J^{r}(\pi)$, where $\pi$ is a real analytic vector bundle corresponding to dependent and independent variables of (5). Hence, in order to complexify the system, one needs first to complexify $J^{r}(\pi)$. Such a construction is described in section 2.1 starting from a given complexification of $\pi$.

We end the paper by applying the above results to the elliptic Euler-Darboux equation

$$
\mathcal{E}_{E D}=\left\{(x+y)\left(u_{x x}+u_{y y}\right)+u_{x}+u_{y}=0\right\}
$$

which appears in two recent papers $([16],[17])$ devoted to the study of Lorentzian Ricci flat 4-metrics with a bidimensional nonabelian Lie algebra $\mathcal{G}$ of Killing vector fields with non null orbits. This equation can be mapped into its hyperbolic analogue

$$
\mathcal{Y}_{E D}=\left\{2(\xi+\eta) v_{\xi \eta}+v_{\xi}+v_{\eta}=0\right\}
$$

already studied in $[13,14,15]$, via a linear contact transformation satisfying the assumptions of theorem 4 . In fact, in proposition 7 we classify all linear contact transformations satisfying such assumptions.

We note that map (8) is not a Lie algebra morphism. However, as the algebra of generalised symmetries of (10) is given in terms of recursion operators, we use theorem 6 to compute the Lie algebra structure of equation (9). 
We would like to stress that our method applies to a large class of differential equations. Just to give a well-known example, think of the minimal surface equation which is mapped, via a linear complex map, into the Born-Infeld equation, whose contact symmetries are known (see [11]).

\section{BASIC NOTIONS ON SYMMETRIES}

In this section we recall the basics about jet bundles and symmetries of partial differential equations (for further details see $[2,7,9,12,20,21]$ ).

Let $M$ be an $n$-dimensional smooth manifold and $\pi: E \rightarrow M$ be a vector bundle, $\operatorname{dim} E=n+m$. Let $\mathcal{U} \subset M$ be a neighbourhood of $M$ such that $\pi^{-1}(\mathcal{U}) \simeq \mathcal{U} \times \mathbb{R}^{m}$ and let $\left(x^{\lambda}, u^{i}\right), \lambda=1 \ldots n, i=1 \ldots m$, with $\left(x^{\lambda}\right)$ coordinates on $\mathcal{U}$, be the corresponding trivialisation. Then a local section of $\pi$ is locally described by $u^{i}=f^{i}\left(x^{1}, x^{2}, \ldots, x^{n}\right)$. We shall denote by $\Gamma_{\text {loc }}(\pi)$ the $C^{\infty}(M)$-module of local sections of $\pi$.

Two local sections $s$ and $\widetilde{s}$ of $\pi$ are said to be $r$-contact equivalent at the point $x \in M$ if their Taylor expansions at this point coincide up to order $r$. This is an equivalence relation, and we shall denote by $[s]_{x}^{r}$ an equivalence class. The set $J^{r}(\pi)$ of all the equivalence classes $[s]_{x}^{r}$ is called the jet bundle of order $r$ and it has a natural vector bundle structure. A chart $\left(x^{\lambda}, u_{\tau}^{i}\right)$ on $J^{r}(\pi)$ is defined by $u_{\tau}^{i}\left([s]_{x}^{r}\right)=\frac{\partial^{|\tau|} f^{i}}{\partial x^{\tau}}(x)$, where $\tau=\left(\tau_{1}, \tau_{2}, \ldots, \tau_{k}\right)$ with $|\tau| \stackrel{\text { def }}{=} k \leqslant r$ and $1 \leqslant \tau_{i} \leqslant n$, is a multi-index and $\frac{\partial^{|\tau|}}{\partial x^{\tau}}$ stands for $\frac{\partial^{|\tau|}}{\partial x^{\tau_{1}} \cdots \partial x^{\tau_{k}}}$.

We have the following natural maps:

1. the embeddings $j_{r} s: M \rightarrow J^{r}(\pi), x \mapsto[s]_{x}^{r}$,

2. the projections $\pi_{k, h}: J^{k}(\pi) \rightarrow J^{h}(\pi),[s]_{x}^{k} \mapsto[s]_{x}^{h} \quad k \geqslant h$,

3. The base projections $\pi_{r}: J^{r}(\pi) \rightarrow M,[s]_{x}^{r} \mapsto x$.

The contact plane $\mathcal{C}_{\theta_{r}}$ at the point $\theta_{r} \in J^{r}(\pi)$ is the span of the planes $T_{\theta_{r}}\left(j_{r} s(M)\right)$, with $s \in \Gamma_{\text {loc }}(\pi)$ varying among sections whose $r$-jet at $\pi_{r}\left(\theta_{r}\right)$ coincides with $\theta_{r}$. We have the contact distribution $\theta_{r} \mapsto \mathcal{C}_{\theta_{r}}$ on $J^{r}(\pi)$. A diffeomorphism of $J^{r}(\pi)$ is called a contact transformation if it is a symmetry of the contact distribution (that is, if it preserves contact planes). A vector field on $J^{r}(\pi)$ whose local flow consists of contact transformations is called a contact field. We note that a point $\theta_{r+1}=[s]_{x}^{r+1}$ of $J^{r+1}(\pi)$. is completely characterised by $T_{\theta_{r}}\left(j_{r} s(M)\right)$ with $\theta_{r}=\pi_{r+1, r}\left(\theta_{r+1}\right)$. Then we can lift a contact transformation $G$ of $J^{r}(\pi)$ to a contact transformation $G^{(1)}$ of $J^{r+1}(\pi)$ by considering $d_{\theta_{r}} G\left(T_{\theta_{r}}\left(j_{r} s(M)\right)\right)$. Of course we can lift contact fields by lifting their local flows. According to a classical result by Lie and Baecklund, any contact transformation is the lifting: 1) of a first order contact transformation if $m=\operatorname{rank} \pi=1$; $)$ of a diffeomorphism of $J^{0}(\pi)=E$ if $m>1$. An analogous result holds for contact fields. 
A differential equation $\mathcal{E}$ of order $r$ is a submanifold of $J^{r}(\pi)$. A linear equation is a linear subbundle of $\pi_{r}: J^{r}(\pi) \rightarrow M$. A (local) solution of $\mathcal{E}$ is a section $s$ of $\pi$ such that $j_{r} s(M) \subset \mathcal{E}$. The 1-prolongation $\mathcal{E}^{(1)}$ of the equation $\mathcal{E}$ is the set of first order "differential consequences" of $\mathcal{E}$. Geometrically:

$$
\mathcal{E}^{(1)}=\left\{[s]_{x}^{r+1} \in J^{r+1}(\pi) \mid s \in \Gamma_{\mathrm{loc}}(\pi),[s]_{x}^{r} \in \mathcal{E}, T_{[s]_{z}^{r}}\left(j_{r} s(M)\right) \subset \mathcal{T}_{[s]_{x}} \mathcal{E}\right\} .
$$

By iteration we can define the $l$-prolongation $\mathcal{E}^{(l)}$. Locally, if the equation $\mathcal{E}$ is described by $\left\{F^{i}=0\right\}$, with $F^{i} \in C^{\infty}\left(J^{r}(\pi)\right)$, then $\mathcal{E}^{(l)}$ is described by $\left\{D_{\tau}\left(F^{i}\right)=0\right\}$ with $0 \leqslant|\tau| \leqslant l$, where $D_{\tau}=D_{\tau_{1}} \circ D_{\tau_{3}} \circ \cdots \circ D_{\tau_{k}}$ and $D_{\lambda}$ are the total derivatives:

$$
D_{\lambda}=\frac{\partial}{\partial x^{\lambda}}+\sum_{j, \tau} u_{\tau, \lambda}^{j} \frac{\partial}{\partial u_{\tau}^{j}} \text {. }
$$

Note that the above definitions of $r$-contact equivalence and $r$-th order jet space make sense even in the case $r=\infty$. Obviously, $J^{\infty}(\pi)$ is not a finite dimensional smooth manifold (its points are sequences of the form $\left\{\theta_{r}\right\}, r \in \mathbb{N}_{0}$, with $\theta_{r} \in J^{r}(\pi)$ and $\left.\pi_{r, r-1}\left(\theta_{r}\right)=\theta_{r-1}\right)$. However, a very rich differential calculus can be developed on it, making it an extremely useful tool in symmetry analysis of partial differential equations as well as in many other fields. Here we limit ourselves to recall just a few basic facts about the differential structure on $J^{\infty}(\pi)$ (for further details see [2]).

By definition, smooth functions on $J^{\infty}(\pi)$ are pullbacks of smooth functions on finite order jet spaces along projections $\pi_{\infty, k}$. Thus, $C^{\infty}\left(J^{\infty}(\pi)\right)$ is a filtered algebra (the degree being the jet order of the pullbacked function). Consequently, vector fields on $J^{\infty}(\pi)$ are defined as derivations $X: C^{\infty}\left(J^{\infty}(\pi)\right) \rightarrow C^{\infty}\left(J^{\infty}(\pi)\right)$ such that $\operatorname{deg} X(f)-\operatorname{deg} f$ is a constant integer depending only on $X$. Vector fields on $J^{\infty}(\pi)$ do not admit, generally, a flow, even locally. For instance, $D_{\lambda}$ is a vector field on $J^{\infty}(\pi)$ with degree 1 . A tangent vector at a point $\theta=\left\{\theta_{r}\right\} \in J^{\infty}(\pi)$ is a sequence $\xi=\left\{\xi_{r}\right\}$ such that $\xi_{r} \in T_{\theta_{r}} J^{r}(\pi)$ and $d_{\theta_{r}} \pi_{r, r-1}\left(\xi_{r}\right)=\xi_{r-1}$. The contact plane $\mathcal{C}_{\theta}$ is the sequence $\left\{\mathcal{C}_{\theta_{r}}\right\}$. Contact distribution $\theta \longmapsto \mathcal{C}_{\theta}$ on $J^{\infty}(\pi)$ is $n$-dimensional (it is spanned by total derivatives $\left\{D_{\lambda}\right\}_{1 \leqslant \lambda \leqslant n}$ ) and integrable, in the sense that its generators satisfy Frobenius conditions. A vector field on $J^{\infty}(\pi)$ lying in the contact distribution $\mathcal{C}$ is called trivial as it is tangent to all integral manifolds of $\mathcal{C}$. Any contact field $X$ on $J^{\infty}(\pi)$ can be splitted in a vertical and a trivial part. More precisely we have that:

$$
X=X_{\varphi}+T
$$

where

$$
X_{\varphi}=\sum_{j, \tau} D_{\tau}\left(\varphi^{j}\right) \frac{\partial}{\partial u_{\tau}^{j}}
$$

with $\varphi=\sum_{j=1}^{m} \varphi^{j} \frac{\partial}{\partial u^{j}}, \varphi^{j} \in C^{\infty}\left(J^{\infty}(\pi)\right)$, and $T$ is a trivial vector field. Fields of the form (11), called evolutionary vector fields, are the only vertical contact fields on $J^{\infty}(\pi) ; \varphi$ is 
called the characteristic (or generating section, [2]) of $X_{\varphi}$. The correspondence $\varphi \leftrightarrow X_{\varphi}$ is one-to-one. Evolutionary vector fields form a Lie subalgebra, isomorphic to the algebra of generating sections with respect to Jacobi bracket

$$
\left\{X_{\varphi}, X_{\psi}\right\}=X_{\{\varphi, \psi\}}
$$

A classical symmetry of $\mathcal{E}$ is a contact field on $J^{r}(\pi)$ tangent to $\mathcal{E}$. If, in particular, it is a lift of a vector field on $E$, then it is called a point symmetry. A contact field on $J^{\infty}(\pi)$ tangent to $\mathcal{E}^{(\infty)}$ is called an external generalised (or higher, [2]) symmetry. A vector field on $\mathcal{E}^{(\infty)}$ which preserves the contact distribution induced on $\mathcal{E}^{(\infty)}$ is called an internal generalised symmetry.

Now we are interested in non-trivial symmetries, that is symmetries of the form $X_{\varphi}$. Locally, if the equation $\mathcal{E}$ is described by the system $\left\{F^{i}=0\right\}$, with $F^{i} \in C^{\infty}\left(J^{r}(\pi)\right)$, then the vector field $X_{\varphi}$ is an external generalised symmetry of $\mathcal{E}$ if and only if $\left.X_{\varphi}\left(F^{i}\right)\right|_{\mathcal{E}^{(\infty)}}=0$. Locally

$$
\sum_{j, \tau} \frac{\partial F^{i}}{\partial u_{\tau}^{j}} \widetilde{D}_{\tau}\left(\widetilde{\varphi}^{j}\right)=0
$$

where the tilde denotes the restriction to $\mathcal{E}^{(\infty)}$.

It is easy to realise that any external generalised symmetry restricts to an internal generalised symmetry. The converse is also true: each internal generalised symmetry can be obtained by restricting on $\mathcal{E}^{(\infty)}$ some external one. For this reason we shall not distinguish them, and we shall call them simply generalised symmetries. Then we shall denote by $\operatorname{Sym}(\mathcal{E})$ the algebra of (non-trivial) generalised symmetries of $\mathcal{E}$.

A vector valued operator $\Delta$ acting on vector functions on $J^{\infty}(\pi)$ is called $\mathcal{C}$. differential if its restriction to $\mathcal{E}^{(\infty)}$ is well defined for any differential equation $\mathcal{E}$. In local coordinates, $\Delta=\left\|\Delta_{i j}\right\|$, where

$$
\Delta_{i j}=\sum_{\boldsymbol{\tau}} a_{i j}^{\tau} D_{\tau}, \quad \text { with } a_{i j}^{\tau} \in C^{\infty}\left(J^{\infty}(\pi)\right)
$$

Finally, a recursion operator $\Re \in \operatorname{Rec}(\mathcal{E})$ for a differential equation $\mathcal{E}$ is a linear $\mathcal{C}$-differential operator which maps $\operatorname{Sym}(\mathcal{E})$ into itself.

\section{SYMMETRIES AND RECURSION OPERATORS OF PARTIAL DIFFERENTIAL EQUATIONS BY COMPLEX TRANSFORMATIONS}

2.1. HOLOMORPHIC EXTENSION OF JET BUNDLES. As we said in the introduction, we consider, instead of a real contact transformation of $J^{r}(\pi)$, a complex one. This implies that the transformation itself is defined not on $J^{r}(\pi)$ but on a complexification of it. Below we construct such a complexification. 
Let us recall the definition of a complexification of a real analytical manifold. Let $M$ be an $n$-dimensional real analytic manifold. A complexification of $M$ is an $n$-dimensional complex manifold $\widehat{M}$ such that:

1. $M$ is a real analytical submanifold of $\widehat{M}$,

2. there exists a conjugation on $\widehat{M}$, that is, an involutive antiholomorphism $\sigma$ such that $M$ is the set of fixed points of $\sigma$.

A $\widehat{M}$ always exists (see [3]). Moreover (see $[8,19]$ ), for any point $x \in M$, there exists a holomorphic chart $\left(z_{1}, \ldots, z_{n}\right)$ defined in a neighbourhood $U$ of $x$ in $\widehat{M}$ such that in it $\sigma$ is represented in the standard way:

$$
\sigma\left(z_{1}, \ldots, z_{n}\right)=\left(\bar{z}_{1}, \ldots, \bar{z}_{n}\right) .
$$

For any holomorphic map $H: \widehat{M} \rightarrow \widehat{M}$, its conjugated map $\bar{H}$ is defined by

$$
\bar{H}=\sigma \circ H \circ \sigma .
$$

Obviously, if $\bar{H}=H$, then $H(M) \subseteq M$.

Let now $\pi: E \rightarrow M$ be a real analytic vector bundle. Then a holomorphic extension of $\pi$ is by definition ([18]) a holomorphic vector bundle $\widehat{\pi}: \widehat{E} \rightarrow \widehat{M}$, where $\widehat{M}$ and $\hat{E}$ are complexification of $M$ and $E$ respectively, and $\widehat{\pi}$ satisfies

1. $\sigma_{\widehat{M}} \circ \widehat{\pi}=\widehat{\pi} \circ \sigma_{\widehat{E}}$

2. $\left.\widehat{\pi}\right|_{E}=\pi$;

3. $\widehat{\pi}$ restricted to $M$ is the complexified vector bundle of $\pi$, with the conjugation on each fibre $\widehat{\pi}^{-1}(a), a \in M$, given by the restriction $\left.\sigma_{\hat{E}}\right|_{\hat{\pi}^{-1}(a)}$;

4. There exists an open covering $\left\{U_{j}\right\}$ of $M$ in $\widehat{M}$ and a corresponding family of trivialisations $\varrho_{j}: \widehat{\pi}^{-1}\left(U_{j}\right) \rightarrow U_{j} \times \mathbb{C}^{m}$ such that

(a) $\sigma_{\hat{E}}\left(\hat{\pi}^{-1}\left(U_{j}\right)\right)=\widehat{\pi}^{-1}\left(U_{j}\right)$;

(b) Let $\theta \in \widehat{\pi}^{-1}(a)$, with $a \in U_{j}$. If $\varrho_{j}(\theta)=(a, w)$, then $\varrho_{j}\left(\sigma_{\widehat{E}}(\theta)\right)$ $=\left(\sigma_{\widehat{M}}(a), \bar{w}\right)$.

The fourth condition simply means that $\sigma_{\hat{E}}$ can be expressed, in an appropriate natural chart on $\widehat{E}$, in the standard way.

It can be proved that, for any real analytical vector bundle $\pi$, there always exists a holomorphic extension of it. Furthermore, the germ along $M$ of such an extension is unique up to holomorphic extension isomorphisms. See [18] for a proof in a more general setting.

Let $\widehat{\pi}$ be a complexification of $\pi$. Then one can consider the complex analytical bundle $J^{k}(\widehat{\pi})$ of $k$-jets of local sections of $\widehat{\pi}$. We want to prove that $J^{k}(\widehat{\pi})$ is a complexification of $J^{k}(\pi)$.

First of all we construct a real analytic immersion $\iota_{k}: J^{k}(\pi) \hookrightarrow J^{k}(\tilde{\pi})$. Namely, let $s \in \Gamma_{\text {loc }}(\pi)$, and $a \in M$. Then $\iota_{k}\left([s]_{a}^{k}\right) \stackrel{\text { def }}{=}[\widehat{s}]_{a}^{k}$, where $\widehat{s} \in \Gamma_{\text {loc }}(\widehat{\pi})$, defined in a conveniently 
small neighbourhood of $a$ in $\widehat{M}$, is a holomorphic extension of $s$. The definition is well posed due to the unicity of the germ of the holomorphic extension of real analytic function at a point (see [5]). We set $\iota=\iota_{\infty}$.

The conjugation $\sigma_{k}$ on $J^{k}(\widehat{\pi})$ is defined by formula $\sigma_{k}\left([s]_{a}^{k}\right)=[\bar{s}]_{\sigma_{\widehat{M}}(a)}^{k}$, with $a \in \widehat{M}$, $s \in \Gamma_{\text {loc }}(\widehat{\pi})$ and $\bar{s}=\sigma_{\widehat{E}} \circ s \circ \sigma_{\widehat{M}}$. Obviously $\sigma_{k}$ is the $k$-contact prolongation of $\sigma_{\widehat{E}}$.

2.2. MAIN THEOREM. In this section it will be shown how symmetries and recursion operators of a system of partial differential equations transform under a complex point transformation. In particular we prove the main result of the paper, namely the isomorphism theorems 4 and 6 .

Let $N$ be a real analytic manifold and $\widehat{N}$ be a complexification. By an entire function on $N$ with respect to $\widehat{N}$ we mean a real analytic function on $N$ which holomorphically extends to the whole $\widehat{N}$. We note that, if such an extension exists, it is unique [6].

Let $\pi: E \rightarrow M$ be a real analytic vector bundle. From now on we fix a complexification $\widehat{\pi}$ of it. We introduce the following definitions and notations. Denote by:

1. $\mathcal{F}\left(J^{r}(\pi)\right)$ the algebra of real-valued functions which are quotient of two entire functions on $J^{r}(\pi)$; note that the definition holds also for $r=\infty$.

2. $\mathcal{F}\left(J^{r}(\widehat{\pi})\right)$ the algebra of holomorphic functions defined on the whole $J^{r}(\widehat{\pi})$;

3. $\mathcal{F}_{\mathbf{C}}\left(J^{r}(\pi)\right) \stackrel{\text { def }}{=}\left\{f_{1}+i f_{2} \mid f_{1}, f_{2} \in \mathcal{F}\left(J^{r}(\pi)\right)\right\}$.

Any function $f / g \in \mathcal{F}\left(J^{r}(\pi)\right)$ can be uniquely extended to $\mathcal{F}\left(J^{r}(\widehat{\pi})\right)$ provided we exclude the set of $J^{r}(\widehat{\pi})$ described by $\hat{g}=0$. Thus, the holomorphic extension operator

$$
\rho: \mathcal{F}_{\mathbf{C}}(J(\pi)) \rightarrow \mathcal{F}(J(\hat{\pi}))
$$

is well defined (we shall occasionally denote $\rho(f)$ by $\widehat{f}$ ). It holds $\overline{\rho(f)}=\rho(\bar{f})$.

Consider the $r$-th order system of partial differential equations $\mathcal{E} \subset J^{r}(\pi)$ locally given by the system

$$
\mathcal{E}=\left\{F^{i}=0\right\}_{i=1, \ldots, s},
$$

with $F^{i} \in \mathcal{F}\left(J^{r}(\dot{\pi})\right)$.

Let $\widehat{\mathcal{E}}$ be the complexification of $\mathcal{E}$, that is, $\widehat{\mathcal{E}}=\left\{\widehat{F^{i}}=0\right\}$, with $\widehat{F^{i}}$ the holomorphic extension of $F^{i}$. Let $h: \widehat{M} \rightarrow \widehat{M}$ be a biholomorphism and $H_{0}: \widehat{E} \rightarrow \widehat{E}$ be an automorphism of $\hat{\pi}$ on $h$. Locally $H_{0}$ is given by formulas:

$$
H_{0}:\left\{\begin{array}{l}
y=h(x) \\
v=A(x) \cdot u
\end{array}\right.
$$

with $(x, u)=\left(x^{\lambda}, u^{j}\right)$ coordinates of a point $p \in \widehat{E},(y, v)=\left(y^{\lambda}, v^{j}\right)$ coordinates of $H_{0}(p)$, and $x \mapsto A(x)$ a holomorphic map from $\widehat{M}$ to $G L(m, \mathbb{C})$. 
Define $\mathcal{Y}$ as follows:

$$
\mathcal{Y}=H_{0}^{(r)}(\widehat{\mathcal{E}}) \cap J(\pi)
$$

Locally,

$$
\mathcal{Y}=\left\{G^{i}=0\right\}_{i=1, \ldots, s}
$$

with $G^{i}=\left.\widehat{F^{i}} \circ\left(H_{0}^{(r)}\right)^{-1}\right|_{J r(\pi)}$. We assume that $\mathcal{Y}$ has the same dimension as $\mathcal{E}$, which is equivalent to the fact that $G^{i}$ are (proportional to) real valued functions. We have that

$$
\mathcal{Y}^{(k)}=H_{0}^{(r+k)}\left(\widehat{\mathcal{E}}^{(k)}\right) \cap J^{r+k}(\pi) .
$$

Also, $\operatorname{dim} \mathcal{E}^{(k)}=\operatorname{dim} \mathcal{Y}^{(k)}$. This can be checked directly by writing down the local expressions of the prolonged equations and computing the ranks of their jacobian.

From now on, we denote by $H: J^{\infty}(\widehat{\pi}) \rightarrow J^{\infty}(\widehat{\pi})$ the infinite contact prolongation $H_{0}^{(\infty)}$ of $H_{0}$.

For each $f \in \mathcal{F}\left(J^{\infty}(\pi)\right)$, we define its "pullback" $\mathcal{H}^{*}$ along $H$ as

$$
\mathcal{H}^{*}(f)=\left.\left(H^{*}(\widehat{f})\right)\right|_{J^{\infty}(\pi)}
$$

that is,

$$
\mathcal{H}^{*}=\iota^{*} \circ H^{*} \circ \rho
$$

Analogously, let $\Delta: \mathcal{F}_{\mathbf{C}}\left(J^{k}(\pi)\right) \rightarrow \mathcal{F}_{\mathbf{C}}\left(J^{\infty}(\pi)\right)$ a differential operator and $\widehat{\Delta}: \mathcal{F}\left(J^{k}(\widehat{\pi})\right)$ $\rightarrow \mathcal{F}\left(J^{\infty}(\widehat{\pi})\right)$, defined by

$$
\widehat{\Delta}=\rho \circ \Delta \circ \iota^{*}
$$

(recall that $\left.\iota^{*}\right|_{\mathcal{F}\left(J^{k}(\hat{\pi})\right)}=\iota_{k}^{*}$ ) be its holomorphic extension. Then the image of $\Delta$ via $H$ is defined as

$$
\mathcal{H}(\Delta)=i \circ H(\widehat{\Delta}) \circ \rho
$$

with

$$
H(\widehat{\Delta})=H^{-1} \circ \widehat{\Delta} \circ H^{*}
$$

or equivalently by

$$
\mathcal{H}(\Delta)=\mathcal{H}^{-1 *} \circ \Delta \circ \mathcal{H}^{*}
$$

Obviously, when $\Delta$ is non-scalar, then (19) is meant componentwise.

We define the conjugated operator $\bar{\Delta}$ of $\Delta$ by

$$
\bar{\Delta}(f)=\overline{\Delta(\bar{f})}
$$

Note that

$$
\bar{H}(\Delta)=\overline{H(\bar{\Delta})} \text {. }
$$

From (18), if $\mathcal{E}^{(\infty)}$ is described by $\left\{D_{\sigma}\left(F^{i}\right)=0\right\}_{|\sigma| \geqslant 0}$, then $\mathcal{Y}^{(\infty)}$ is described by $\left\{\mathcal{H}^{-1 *}\left(D_{\sigma}\left(F^{i}\right)\right)=0\right\}|\sigma| \geqslant 0$. 
Proposition 1. The following identities hold:

1. $\widehat{\mathcal{H}^{*}(f)}=H^{*}(\widehat{f})$ for any $f \in \mathcal{F}_{\mathrm{C}}\left(J^{\infty}(\pi)\right)$;

2. $\widehat{\mathcal{H}(\Delta)}=H(\widehat{\Delta})$ for any differential operator $\Delta: \mathcal{F}_{\mathbf{C}}\left(J^{k}(\pi)\right) \rightarrow \mathcal{F}_{\mathbf{C}}\left(J^{\infty}(\pi)\right)$.

Proof: Both identities easily follow from the uniqueness of the holomorphic extension of elements of $\mathcal{F}_{\mathbf{C}}\left(J^{\infty}(\pi)\right)$.

We associate with $\Delta$ its real part $\Delta_{1}$ and its imaginary part $\Delta_{2}$, defined by

$$
\Delta_{j}(f)=(\Delta(f))_{j}
$$

for any $f \in \mathcal{F}_{\mathbf{C}}\left(J^{\infty}(\pi)\right), j=1,2$.

In the last part of this section we prove the main result of this paper. Namely, we construct (see theorem 4) a vector space isomorphism $\theta$ from the algebra $\operatorname{Sym}_{\mathcal{F}}(\mathcal{E})$ of generalised symmetries of $\mathcal{E}$ with components in $\mathcal{F}\left(J^{\infty}(\pi)\right)$ into that of $\mathcal{Y}$, which we denote by $\operatorname{Sym}_{\mathcal{F}}(\mathcal{Y})$.

In order to prove theorem 4 some technical lemmas are necessary.

First of all, a straightforward computation shows that, for any vector field $X$ : $\mathcal{F}\left(J^{\infty}(\pi)\right) \rightarrow \mathcal{F}\left(J^{\infty}(\pi)\right)$, the restrictions of $\left(\mathcal{H}^{-1}(X)\right)_{1}$ and $\left(\mathcal{H}^{-1}(X)\right)_{2}$ to $\mathcal{F}\left(J^{\infty}(\pi)\right)$ are vector fields on $J^{\infty}(\pi)$.

LEMMA 2. Let $\varphi: \mathcal{F}\left(J^{0}(\pi)\right) \rightarrow \mathcal{F}\left(J^{\infty}(\pi)\right)$ be the characteristic of the evolutionary vector field $X_{\varphi}$. Then we have that

$$
\mathcal{H}^{-1}\left(X_{\varphi}\right)=X_{\mathcal{H}^{-1}(\varphi)} \stackrel{\text { def }}{=} X_{\left(\mathcal{H}^{-1}(\varphi)\right)_{1}}+i X_{\left(\mathcal{K}^{-1}(\varphi)\right)_{2}}
$$

ProOF: First of all, $\widehat{X}_{\varphi}=X_{\widehat{\varphi}}$ is an evolutionary field on $J^{\infty}(\widehat{\pi})$. Then its image $H^{-1}\left(X_{\hat{\varphi}}\right)$ is a contact field. Moreover it is vertical; in fact, due to the block form of $H$, the function $H^{-1 *}\left(\widehat{x}_{\lambda}\right)$ depends only on $\widehat{x^{1}} \ldots \widehat{x^{n}}$ and, hence, $X_{\widehat{\varphi}}$ vanishes on it. Therefore, if $B(x)$ is the inverse matrix of $A(x)$ in (15), $H^{-1}\left(X_{\hat{\varphi}}\right)=X_{\widehat{\psi}}$ with

$$
\psi=\sum_{j=1}^{m} \mathcal{H}^{-1}\left(X_{\varphi}\right)\left(u^{j}\right) \frac{\partial}{\partial u^{j}}=\sum_{j=1}^{m} \mathcal{H}^{*}\left(\sum_{h=1}^{m} B_{h}^{j}(x) \varphi^{h}\right) \frac{\partial}{\partial \omega^{j}}=\mathcal{H}^{-1}\left(\sum_{h=1}^{m} \varphi^{h} \frac{\partial}{\partial u^{h}}\right)
$$

From now on we identify vector field $X_{\varphi}$ with its characteristic $\varphi$.

LEMMA 3. If $\varphi \in \operatorname{Sym}_{\mathcal{F}}(\mathcal{Y})$ then $\left(\mathcal{H}^{-1}(\varphi)\right)_{1}$ and $\left(\mathcal{H}^{-1}(\varphi)\right)_{2}$ belong to $\operatorname{Sym}_{\mathcal{F}}(\mathcal{E})$.

PROOF: By hypothesis, we have that

$$
X_{\varphi}\left(\mathcal{H}^{-1 *}\left(D_{\sigma}\left(F^{j}\right)\right)\right)=\sum_{|\tau| \geqslant 0} \sum_{i=1}^{B} a_{\sigma i}^{\tau j} \mathcal{H}^{-1 *}\left(D_{\tau}\left(F^{i}\right)\right)
$$

which implies

$$
\mathcal{H}^{*}\left(X_{\varphi}\left(\mathcal{H}^{-1 *}\left(D_{\sigma}\left(F^{j}\right)\right)\right)\right)=\sum_{|\tau| \geqslant 0} \sum_{i=1}^{s} \mathcal{H}^{*}\left(a_{\sigma i}^{\tau j}\right) D_{\tau}\left(F^{i}\right)
$$


that is the left hand side term of the above expression vanishes on $\mathcal{E}^{(\infty)}$. The lemma follows taking into account that $\mathcal{H}^{*} \circ X_{\varphi} \circ \mathcal{H}^{-1 *}=\mathcal{H}^{-1}\left(X_{\varphi}\right)$ and in view of previous lemma.

Now it is possible to prove the following

THEOREM 4 . Let $H_{0}: J^{0}(\widehat{\pi}) \rightarrow J^{0}(\hat{\pi})$ be such that

$$
\overline{H_{0}} \circ H_{0}^{-1}=H_{0} \circ{\overline{H_{0}}}^{-1} \text {. }
$$

Then the linear map

$$
\Theta: \operatorname{Sym}_{\mathcal{F}}(\mathcal{Y}) \rightarrow \operatorname{Sym}_{\mathcal{F}}(\mathcal{E}), \varphi \mapsto\left(\mathcal{H}^{-1}(\varphi)\right)_{1}+\left(\mathcal{H}^{-1}(\varphi)\right)_{2}
$$

is a vector space isomorphism.

Proof: First of all, note that (21) implies that $\overline{H_{0}} \circ H_{0}^{-1}$ maps the total space $E$ of $\pi$ into itself, or, equivalently, $\overline{H_{0}}(E)=H_{0}(E)$. Moreover, if $H_{0}$ satisfies (21) then also $H_{0}^{(k)}$ does for any $k=0,1, \ldots \infty$. This comes from the fact that $\sigma_{k}=\sigma_{\bar{E}}^{(k)}$.

Now, let $\varphi \in \mathcal{F}\left(J^{\infty}(\pi)\right)$. Then

$$
\begin{aligned}
& \left(\mathcal{H}\left(\mathcal{H}^{-1}(\varphi)\right)_{1}\right)_{1}-\left(\mathcal{H}\left(\mathcal{H}^{-1}(\varphi)\right)_{2}\right)_{2}=\varphi \\
& \left(\mathcal{H}\left(\mathcal{H}^{-1}(\varphi)\right)_{1}\right)_{2}=\left(\mathcal{H}\left(\mathcal{H}^{-1}(\varphi)\right)_{2}\right)_{1}=0
\end{aligned}
$$

In fact,

$$
\begin{aligned}
\varphi & =\mathcal{H}\left(\mathcal{H}^{-1}(\varphi)\right)=\mathcal{H}\left(\left(\mathcal{H}^{-1}(\varphi)\right)_{1}+i\left(\mathcal{H}^{-1}(\varphi)\right)_{2}\right)=\mathcal{H}\left(\left(\mathcal{H}^{-1}(\varphi)\right)_{1}\right)+i \mathcal{H}\left(\left(\mathcal{H}^{-1}(\varphi)\right)_{2}\right) \\
& =\left(\mathcal{H}\left(\mathcal{H}^{-1}(\varphi)\right)_{1}\right)_{1}-\left(\mathcal{H}\left(\mathcal{H}^{-1}(\varphi)\right)_{2}\right)_{2}+i\left(\left(\mathcal{H}\left(\mathcal{H}^{-1}(\varphi)\right)_{1}\right)_{2}+\left(\mathcal{H}\left(\mathcal{H}^{-1}(\varphi)\right)_{2}\right)_{1}\right) .
\end{aligned}
$$

As $\varphi$ is a real function, equation (22) and

$$
\left(\mathcal{H}\left(\mathcal{H}^{-1}(\varphi)\right)_{1}\right)_{2}+\left(\mathcal{H}\left(\mathcal{H}^{-1}(\varphi)\right)_{2}\right)_{1}=0
$$

hold. Also, $\mathcal{H}\left(\overline{\mathcal{H}^{-1}(\varphi)}\right)$ is real. In fact

$$
\overline{\mathcal{H}^{-1}(\varphi)}=\overline{\mathcal{H}^{-1}}(\varphi),
$$

and then

$$
\mathcal{H}\left(\overline{\mathcal{H}^{-1}(\varphi)}\right)=\mathcal{H}\left(\overline{\mathcal{H}^{-1}}(\varphi)\right)=\left.\left(H \circ \bar{H}^{-1}\right)^{*}(\widehat{\varphi})\right|_{J^{\infty}(\pi)},
$$

which, keeping in mind that $H \circ \bar{H}^{-1}$ is a real transformation, proves the reality of $\mathcal{H}\left(\overline{\mathcal{H}^{-1}(\varphi)}\right)$. This implies that

$$
\left(\mathcal{H}\left(\mathcal{H}^{-1}(\varphi)\right)_{1}\right)_{2}-\left(\mathcal{H}\left(\mathcal{H}^{-1}(\varphi)\right)_{2}\right)_{1}=0
$$


which, together with (24), gives equation (23).

Finally, the map $\Theta^{\prime}: \eta \mapsto(\mathcal{H}(\eta))_{1}-(\mathcal{H}(\eta))_{2}$ is both the left and right inverse map of $\theta$. In fact

$$
\Theta^{\prime}(\Theta(\varphi))=\left(\mathcal{H}\left(\mathcal{H}^{-1}(\varphi)\right)_{1}\right)_{1}+\left(\mathcal{H}\left(\mathcal{H}^{-1}(\varphi)\right)_{1}\right)_{2}-\left(\mathcal{H}\left(\mathcal{H}^{-1}(\varphi)\right)_{2}\right)_{1}-\left(\mathcal{H}\left(\mathcal{H}^{-1}(\varphi)\right)_{2}\right)_{2}
$$

which is equal to $\varphi$ in view of equations (22) and (23). The same reasoning holds for $\theta\left(\Theta^{\prime}(\eta)\right)$. Theorem is proved.

REMARK 5. The map $\theta$ is not a Lie algebra morphism, as a direct computation shows.

Denote by $\operatorname{Rec}_{\mathcal{F}}(\mathcal{Y})$ and $\operatorname{Rec}_{\mathcal{F}}(\mathcal{E})$, respectively, the Lie algebra of recursion operators of $\mathcal{Y}$ and $\mathcal{E}$ with coefficients in $\mathcal{F}\left(J^{\infty}(\pi)\right)$. The same reasonings used in the proof of theorem 4 lead to the following

THEOREM 6. The linear map

$$
\Psi: \operatorname{Rec}_{\mathcal{F}}(\mathcal{Y}) \rightarrow \operatorname{Rec}_{\mathcal{F}}(\mathcal{E}), \Re \mapsto\left(\mathcal{H}^{-1}(\Re)\right)_{1}+\left(\mathcal{H}^{-1}(\Re)\right)_{2}
$$

is a vector space isomorphism.

The latter result will be used in the next section to describe the Lie structure of generalised symmetries of (9).

\section{AN APPLICATION: COMPLEX LINEAR CONTACT TRANSFORMATIONS AND THE ELLIPTIC EULER-DARBOUX EQUATION}

Below we apply the above results to computing generalised symmetries of the elliptic Euler-Darboux equation (9) and determining their Lie algebra structure. As we anticipated in the introduction, we map such equation into its hyperbolic analogue, already studied in $[13,14,15]$, via a complex linear contact transformation. For this reason, we start by determining the most general transformation of this kind satisfying condition (21).

3.1. LiNEAR CONTACT TRANSFORMATIONS. As we are interested in local symmetries, we can consider equation (9) as a submanifold of $J^{2}(\pi)$ where $\pi: \mathbb{R}^{2} \times \mathbf{R} \rightarrow \mathbb{R}^{2}$ is the trivial bundle. In this case $H_{0}$ acts on the natural complexification $\widehat{\pi}: \mathbb{C}^{2} \times \mathbb{C} \rightarrow \mathbb{C}^{2}$. In local coordinates $(x, y, u)$ on $\mathbb{C}^{2} \times \mathbf{C}, H_{0}$ is described by

$$
H_{0}:\left\{\begin{array}{l}
\xi=h_{11} x+h_{12} y \\
\eta=h_{21} x+h_{22} y \\
v=a(x, y) u
\end{array}\right.
$$

with $h=\left\|h_{i j}\right\|$ a complex invertible matrix, and $a: \mathbb{C}^{2} \rightarrow \mathbb{C}$ holomorphic. 
Proposition 7. Let $h=A+i B$ with $A, B$ real matrices. Then, excluding the trivial cases $A=0$ or $B=0$, transformation (26) satisfies condition (21) if and only if

1. $\operatorname{det} A=\operatorname{det} B=0$;

2. $a(x, y) a\left(h \cdot h^{-1} \cdot(x, y)^{T}\right)$ is a real constant function on $\mathbb{C}^{2}$.

Moreover, $h$ is similar, via a real matrix, to a matrix of the form $P \cdot G$, where $G$ is a real matrix and

$$
P=\left(\begin{array}{cc}
1 & i \\
1 & -i
\end{array}\right)
$$

Proof: Let $h^{-1}=X+i Y$, with $X, Y$ real matrices. Then it is easily checked that $\bar{h} \circ h^{-1}$ is real if and only if

$$
A Y=B X=0
$$

or, equivalently

$$
Y A=X B=0
$$

which immediately entail the degeneracy of $A$ and $B$. The particular form of matrix $h$ is obtained by straightforward computations which we omit.

To prove the second point note that condition (21) implies that $a(x, y)$ $a\left(h \cdot h^{-1} \cdot(x, y)^{T}\right)$ is real for any $(x, y) \in \mathbb{C}^{2}$. But the only real-valued holomorphic functions are constants.

As, due to the above proposition, matrix $P$ always appears in transformation (26), below we explicitly compute the infinite prolongation of (26) in the case $h=P$ and $a(x, y)=1$. Hence, $H$ can be identified with the infinite dimensional block matrix

$$
H=\left(\begin{array}{ccccccc}
P & & & & & & \\
& 1 & & & & & \\
& & P^{(1)} & & & & \\
& & P^{(2)} & & & \\
& & & \ddots & & \\
& & & & P^{(k)} & \\
& & & & & \ddots
\end{array}\right),
$$

where $P^{(k)}$ is given by

$$
V^{(k)}=P^{(k)} U^{(k)},
$$

with $V^{(k)}=\left(v_{k \xi}, v_{(k-1) \xi, \eta}, \ldots, v_{k \eta}\right)^{T}, U^{(k)}=\left(u_{k x}, u_{(k-1) x, y}, \ldots, u_{k y}\right)^{T}, P^{(k)}=\left\|p_{r s}^{k}\right\|_{r, s=0, \ldots, k}$.

A straightforward computation shows that

$$
p_{r s}^{k}=\frac{1}{s !(k-s) !} D_{(k-r) \xi, r \eta}\left(x^{k-s} y^{s}\right)
$$


By expanding the total derivatives one gets:

$$
\begin{aligned}
p_{r s}^{k} & =(-1)^{k-r} \frac{r !(k-r) !}{2^{k} s !(k-s) !} i^{s} \sum_{\alpha=M(k, r, s)}^{m(k, r, s)}(-1)^{\alpha}\left(\begin{array}{c}
k-s \\
\alpha
\end{array}\right)\left(\begin{array}{c}
s \\
k-r-\alpha
\end{array}\right) \\
& =(-1)^{k-r} \frac{r !(k-r) !}{2^{k}} i^{s} \sum_{\alpha=M(k, r, s)}^{m(k, r, s)} \frac{(-1)^{\alpha}}{\alpha !(k-s-\alpha) !(k-r-\alpha) !(r+s+\alpha-k) !}
\end{aligned}
$$

with $M(k, r, s)=\max (0, k-r-s), m(k, r, s)=\min (k-r, k-s)$.

Let us point out, omitting the proofs, some remarkable properties of matrix $H$.

1. The inverse of block $P^{(k)}$ is $P^{(k)^{-1}}=\left\|q_{r a}^{k}\right\|$, with

$$
q_{r s}^{k}=2^{k} i^{r+s} p_{r s}^{k} .
$$

2. For each block $P^{(k)}$ the following symmetry property holds

$$
\overline{p_{r q}^{k}}=p_{k-r, q}^{k}
$$

for $r, q=0, \ldots, k$.

3. For any $k, \overline{P^{(k)}} \cdot P^{(k)^{-1}}$ is real. This is a special case of the general observation contained in the proof of theorem 4.

4. The following recurrence formulas hold:

$$
\begin{aligned}
p_{r, s}^{k+1} & =\frac{1}{2}\left(p_{r, s}^{k}-i p_{r, s-1}^{k}\right) \quad \text { for } r \leqslant k \\
p_{k+1, s}^{k+1} & =\frac{1}{2}\left(p_{k, s}^{k}+i p_{k, s-1}^{k}\right),
\end{aligned}
$$

where we pose $p_{r,-1}^{k}=p_{r, k+1}^{k}=0$ for $r=0,1, \ldots k$.

3.2. The symmetry algebra of elliptic Euler-DarbouX Equation. Below we apply the results obtained in the previous sections to equation (9). Here $H_{0}=K_{0} \cdot G_{0}$, where

$$
K_{0}=\left(\begin{array}{ll}
P & 0 \\
0 & 1
\end{array}\right) \quad, \quad G_{0}=\left(\begin{array}{ll}
G & 0 \\
0 & 1
\end{array}\right)
$$

with $P$ is the matrix (27) and

$$
G=\left(\begin{array}{cc}
1 / 2 & 1 / 2 \\
1 / 2 & -1 / 2
\end{array}\right)
$$

(see also proposition 7). A direct computation shows that $H_{0}^{(2)}$ transforms equation (9) into equation (10). We recall that $H=H_{0}^{(\infty)}$. 
We choose $\left(x, y, u, u_{x}, u_{y}, \ldots, u_{x,(k-1) y}, u_{k y}, \ldots\right)$ as internal coordinates on $\mathcal{E}_{E D}^{(\infty)}$ and $\left(x, y, u, u_{x}, u_{y}, \ldots, u_{k x}, u_{k y}, \ldots\right)$ as internal coordinates on $\mathcal{Y}_{E D}^{(\infty)}$ (in this case $v=u$ ). However, in order to distinguish the two sets of coordinates, below we denote the latter ones by $\left(\xi, \eta, u, u_{\xi}, u_{\eta}, \ldots, u_{k \xi}, u_{k \eta}, \ldots\right)$. Let $\varphi \in \mathcal{F}\left(J^{\infty}(\pi)\right)$. By (19) we have that $\mathcal{H}^{-1}\left(\varphi \frac{\partial}{\partial u}\right)=\mathcal{H}^{*}(\varphi) \frac{\partial}{\partial u}$. From now on we identify a characteristic of a symmetry with its component.

As an example of computation of symmetries of $\mathcal{E}_{E D}$ we give the following

Proposition 8. Symmetries belonging to $\operatorname{Sym}_{\mathcal{F}}\left(\mathcal{E}_{E D}\right)$ depending on derivatives up to second order are (up to arbitrary solutions of $\mathcal{E}_{E D}$ ) linearly generated by the following ones:

$$
\begin{aligned}
& \phi_{1}=\left(u_{x}+u_{y}+2 u_{x y} x+2 u_{x y} y\right) /(x+y) \\
& \phi_{2}=\left(-y u_{x}+3 x u_{y}-2 u_{x y} y^{2}+2 u_{x y} x^{2}+2 u_{y y} x^{2}+4 u_{y y} y x\right. \\
& \left.+2 u_{y y} y^{2}+x u_{x}+y u_{y}\right) /(x+y) \\
& \phi_{3}=\left(-u_{x} y x-u_{y} y^{2}+u_{y} x^{2}-u_{y} y x-2 y x^{2} u_{x y}-2 y^{2} x u_{x y}+u_{y y} x^{3}\right. \\
& \left.+u_{y y} x^{2} y-u_{y y} x y^{2}-u_{y y} y^{3}\right) /(x+y) \\
& \phi_{4}=\left(3 x^{2} y u_{x}+x^{3} u_{x}-3 y^{3} u_{y}+u y^{2}-u x^{2}+9 x^{2} y u_{y}-3 x y^{2} u_{x}+8 u_{x y} x^{3} y\right. \\
& +4 u_{y y} x^{3} y+4 u_{y y} y^{3} x-8 u_{x y} y^{3} x+12 u_{y y} x^{2} y^{2}+2 u_{x y} x^{4}-2 u_{x y} y^{4} \\
& \left.-2 u_{y y} x^{4}-2 u_{y y} y^{4}+3 x y^{2} u_{y}-x^{3} u_{y}-y^{3} u_{x}\right) /(x+y) \\
& \phi_{5}=\left(u y^{3}+u x^{3}-12 u_{x y} x^{2} y^{3}-12 u_{x y} x^{3} y^{2}-20 u_{y} y^{3} x+12 u_{y} x^{3} y\right. \\
& -18 u_{y} x^{2} y^{2}+4 u_{x} y^{3} x-12 u_{x} x^{3} y-18 u_{x} x^{2} y^{2}-5 u x y^{2}-5 u x^{2} y \\
& +u_{y} y^{4}+5 u_{y} x^{4}+u_{x} x^{4}+5 u_{x} y^{4}-8 u_{y y} y^{4} x-8 u_{y y} y^{3} x^{2}+8 u_{y y} y^{2} x^{3} \\
& \left.+8 u_{y y} y x^{4}+2 u_{x y} y^{4} x+2 u_{x y} x^{4} y+2 u_{x y} x^{5}+2 u_{x y} y^{5}\right) /(x+y) \\
& \phi_{6}=-u_{x}+u_{y} \\
& \phi_{7}=u+2 x u_{x}+2 y u_{y} \\
& \phi_{8}=u x-u y-u_{x} y^{2}+u_{x} x^{2}-2 u_{x} y x+u_{y} x^{2}-u_{y} y^{2}+2 u_{y} y x \\
& \phi_{9}=u
\end{aligned}
$$

ProOF: Firstly we take symmetries of $\mathcal{Y}_{E D}$ depending on second derivatives, obtained in [14], and then we transform them by the map $\theta$ (see theorem 4) and by using formulas (30).

Symmetries $\phi_{6}, \phi_{7}, \phi_{8}$ and $\phi_{9}$ generate the algebra of classical symmetries of $\mathcal{E}_{E D}$. We note that they are point symmetries, as their characteristic are linear in the first derivatives. This means that the corresponding vector fields on $J^{2}(\pi)$ are prolongations of vector fields on $E$ rather than on $J^{1}(\pi)$, or, in other words, that there are no proper contact symmetries. This fact was noticed in [4], where variational aspects of $\mathcal{E}_{E D}$ were also studied. 
In $[13,14,15]$ it is proved that the most general characteristic of a contact symmetry of $\mathcal{Y}_{E D}$ is, up to arbitrary solutions of $\mathcal{Y}_{E D}$, of the following form:

$$
\varphi=\left(c_{1} \frac{(\eta-\xi)}{2}+c_{4}\right) u+\left(-c_{1} \xi^{2}+c_{2} \xi-c_{3}\right) u_{\xi}+\left(c_{1} \eta^{2}+c_{2} \eta+c_{3}\right) u_{\eta}
$$

where $c_{1}, c_{2}, c_{3}, c_{4}$ are arbitrary constants. Since $\mathcal{Y}_{E D}$ is linear, such symmetries determine recursion operators as they are linear in $u$ and in its derivatives ([9]). In particular

$$
\square=D_{\xi}-D_{\eta}, \quad \sigma=\xi D_{\xi}+\eta D_{\eta}+\frac{1}{2}, \quad \tau=\xi^{2} D_{\xi}-\eta^{2} D_{\eta}+\frac{\xi-\eta}{2} \mathbb{I}
$$

are recursion operators. Since generalised symmetries of $\mathcal{Y}_{E D}$ are linear in $u$ and in its derivatives (see theorem 9 below), then in this case the theory of generalised symmetries can be developed using recursion operators as fundamental objects ([9]). For instance, it is natural to ask if by applying arbitrary compositions of (33) to the symmetry $u$ we get the whole algebra of generalised symmetries. For this purpose, let us consider

$$
\square_{j}^{m}=\left[\ldots[\square^{m}, \underbrace{\tau], \ldots, \tau}_{j \text {-times }}]\right.
$$

and

$$
\varphi_{0}=\square(u), \quad \varphi_{1}=\tau(u), \quad \varphi_{2}=\xi u_{\xi \xi}-\eta u_{\eta}+\frac{\xi u_{\xi}-\eta u_{\eta}}{\xi+\eta}
$$

Since the recursion operators (33) are $\mathcal{C}$-differential operators, it is well defined the restriction $\widetilde{\square}_{j}^{m}$ of (34) on the equation. The following three theorems are due to Shemarulin (see $[13,14,15])$.

THEOREM 9 . Let $\varphi \in C^{\infty}\left(\mathcal{Y}_{E D}^{(n-2)}\right)$ be a symmetry of $\mathcal{Y}_{E D}$. Then $\varphi$ has the following form:

$$
\varphi=\phi+\sum_{0 \leqslant k \leqslant n} \mathcal{P}^{k} u_{k \xi}+\sum_{0 \leqslant h \leqslant n} \mathcal{Q}^{h} u_{h \eta},
$$

where $\phi$ is a solution of $\mathcal{Y}_{E D}$ and $\mathcal{P}^{k}$ and $\mathcal{Q}^{h}$ are rational functions in $\xi$ and $\eta$.

Theorem 10. The algebra $\operatorname{Sym}\left(\mathcal{Y}_{E D}\right)$ is the semi-direct sum $A \oplus \operatorname{NSym}\left(\mathcal{Y}_{E D}\right)$ where $A$ is the Abelian infinite dimensional ideal of solutions of $\mathcal{Y}_{E D}$ and $\operatorname{NSym}\left(\mathcal{Y}_{E D}\right)$ is the algebra linearly generated by $u$ and $\varphi_{j}^{m}$ where

$$
\varphi_{j}^{m}=\left\{\ldots \left\{\{\ldots\{\varphi_{0}, \underbrace{\left.\varphi_{2}\right\} \ldots \varphi_{2}}_{(j-1) \text {-times }}\} \underbrace{\left.\varphi_{1}\right\} \ldots \varphi_{1}}_{\text {m-times }}\} .\right.\right.
$$

THEOREM 11. The algebra NSym $\left(\mathcal{Y}_{E D}\right)$ is linearly generated by $u$ and $\widetilde{\square}_{j}^{m}(u)$. Moreover, we have the following relations:

$$
\begin{array}{llrl}
{\left[\square_{j}^{m}, \square\right]} & =-j(2 m-j+1) \square_{j-1}^{m}, & & 1 \leqslant j \leqslant 2 m ; \\
{\left[\square_{j}^{m}, \sigma\right]} & =(m-j) \square_{j}^{m}, & & 0 \leqslant j \leqslant 2 m ; \\
{\left[\square_{j}^{m}, \tau\right]=\square_{j+1}^{m},} & & 0 \leqslant j \leqslant 2 m ;
\end{array}
$$


and

$$
\begin{array}{ll}
\tilde{\square}_{j}^{m}(u) \neq 0, & 0 \leqslant j \leqslant 2 m ; \\
\tilde{\square}_{j}^{m}(u)=0, & j \geqslant 2 m+1 .
\end{array}
$$

with $\widetilde{\square}_{j}^{m}=\left.\square_{j}^{m}\right|_{\mathcal{Y}_{E D}^{(\infty)}}$.

Now we shall reproduce, for the equation $\mathcal{E}_{E D}$, similar results by using the complex transformation $H$.

PROpOSITION 12. The functions belonging to $\operatorname{Sym}_{\mathcal{F}}\left(\mathcal{E}_{E D}\right)$ are linear in the internal jet variables.

Proof: In view of theorems 4 and $9, \operatorname{Sym} \mathcal{F}\left(\mathcal{E}_{E D}\right)$ is formed by images through $\mathcal{H}^{*}$ of symmetries of $\mathcal{Y}_{E D}$. In view of the fact $H$ is a block matrix, the proposition follows if we show that the restriction of $u_{m x, n y}$ to $\mathcal{E}_{E D}^{(m+n-2)}$ is a linear function in the internal jet variables. We show it by induction. Firstly, a straightforward computation shows that

$$
\left.u_{x x, h y}\right|_{\mathcal{E}_{E D}^{(h)}}=-\sum_{k=0}^{h}\left(\begin{array}{l}
h \\
k
\end{array}\right) \frac{(-1)^{k} k !}{(x+y)^{k+1}}\left(u_{x,(h-k) y}+u_{(h-k+1) y}\right)-u_{(h+2) y} .
$$

Now let us suppose that $\left.u_{(m-1) x, n y}\right|_{\mathcal{E}_{E D}^{(m+n-3)}}$ is linear in the internal jet variables. Namely

$$
\left.u_{(m-1) x, n y}\right|_{\mathcal{E}_{E D}^{(m+n-3)}}=\sum_{h=0}^{m+n-2} a^{h} u_{x, h y}+\sum_{j=0}^{m+n-1} b^{j} u_{j y},
$$

where $a^{h}$ and $b^{j}$ are functions of $x$ and $y$. Then

$$
\begin{aligned}
\left.u_{m x, n y}\right|_{\mathcal{E}_{E D}^{(m+n-2)}} & =\widetilde{D}_{x}\left(\left.u_{(m-1) x, n y}\right|_{\mathcal{E}_{E D}^{(m+n-1)}}\right) \\
& =\sum_{h=0}^{m+n-2}\left(\frac{\partial a^{h}}{\partial x} u_{x, h y}+\left.a^{h} u_{x x, h y}\right|_{\mathcal{E}_{E D}^{(h)}}\right)+\sum_{j=0}^{m+n-1}\left(\frac{\partial b^{j}}{\partial x} u_{j y}+b^{j} u_{x, j y}\right) .
\end{aligned}
$$

The assertion is proved in view of (36).

We define the algebra $\operatorname{NSym}\left(\mathcal{E}_{E D}\right)$ as the algebra of generalised symmetries in $\operatorname{Sym}_{\mathcal{F}}\left(\mathcal{E}_{E D}\right)$ up to solutions of $\mathcal{E}_{E D}$. As we have noticed in remark 5 , the map $\theta$ is not a Lie algebra morphism. Anyway we have the following

Proposition 13. The algebra $\operatorname{NSym}\left(\mathcal{E}_{E D}\right)$ is infinite dimensional as vector space and finitely generated as Lie algebra. More precisely

$$
\varrho_{j}^{m}=\left\{\ldots \left\{\{\ldots\{\varrho_{0}, \underbrace{\left.\varrho_{2}\right\} \ldots \varrho_{2}}_{(j-1) \text {-times }}\} \underbrace{\left.\varrho_{1}\right\} \ldots \varrho_{1}}_{m \text {-times }}\}\right.\right.
$$


where

$$
\begin{aligned}
& \varrho_{0}=-u_{x}+u_{y} \\
& \varrho_{1}=\frac{1}{2}\left(x^{2}-2 x y-y^{2}\right) u_{x}+\frac{1}{2}\left(x^{2}+2 x y-y^{2}\right) u_{y}+\frac{1}{2}(x-y) u \\
& \varrho_{2}=(x+y) u_{y y}+(x-y) u_{x y}+\frac{1}{2(x+y)}\left((x-y) u_{x}+(3 x+y) u_{y}\right)
\end{aligned}
$$

form, together with $u$, a linear basis of $\operatorname{NSym}\left(\mathcal{E}_{E D}\right)$.

Proof: The proposition follows taking into consideration that $\Theta$ restricts to $\operatorname{NSym}\left(\mathcal{Y}_{E D}\right)$ and $\operatorname{NSym}\left(\mathcal{E}_{E D}\right)$, theorem 10 , and finally that $\mathcal{H}^{*}\left(\varphi_{0}\right)=i \varrho_{0}, \mathcal{H}^{*}\left(\varphi_{1}\right)=i \varrho_{1}$, $\mathcal{H}^{*}\left(\varphi_{2}\right)=i \varrho_{2}$.

By transforming recursion operators (33) through $\mathcal{H}^{-1}$, we get the following:

Proposition 14.

$$
\mathcal{H}^{-1}(\square)=i \check{\square}, \quad \mathcal{H}^{-1}(\tau)=i \check{\tau}, \quad \mathcal{H}^{-1}(\sigma)=\check{\sigma},
$$

where

$$
\begin{aligned}
\check{\square} & =-D_{x}+D_{y} \\
\check{\tau} & =\left(\frac{1}{2} x^{2}-x y-\frac{1}{2} y^{2}\right) D_{x}+\left(12 x^{2}+x y-\frac{1}{2} y^{2}\right) D_{y}+\frac{1}{2}(x-y) \mathbb{I} \\
\check{\sigma} & =x D_{x}+y D_{y}+\frac{\mathbb{I}}{2}
\end{aligned}
$$

Now, if we define

$$
\nabla_{j}^{m}=\left[\ldots[\check{\square}^{m}, \underbrace{\check{\tau}], \ldots, \tilde{\tau}}_{\text {j-times }}]\right.
$$

we get the Lie structure of $\operatorname{NSym}\left(\mathcal{E}_{E D}\right)$ by means of the following

THEOREM 15. The algebra $\operatorname{NSym}\left(\mathcal{E}_{E D}\right)$ is linearly generated by $u$ and $\tilde{\nabla}_{j}^{m}(u)$. Moreover, we have the following relations:

$$
\begin{array}{lll}
{\left[\nabla_{j}^{m}, \check{\square}\right]=j(2 m-j+1) \nabla_{j-1}^{m},} & & 1 \leqslant j \leqslant 2 m ; \\
{\left[\nabla_{j}^{m}, \check{\sigma}\right]=(m-j) \nabla_{j}^{m},} & & 0 \leqslant j \leqslant 2 m ; \\
{\left[\nabla_{j}^{m}, \check{\tau}\right]=\nabla_{j+1}^{m},} & 0 \leqslant j \leqslant 2 m ;
\end{array}
$$

and

$$
\begin{array}{ll}
\tilde{\nabla}_{j}^{m}(u) \neq 0, & 0 \leqslant j \leqslant 2 m \\
\tilde{\nabla}_{j}^{m}(u)=0, & j \geqslant 2 m+1 .
\end{array}
$$


Proof: We have that

$$
\begin{aligned}
\mathcal{H}^{-1}\left(\square_{j}^{m}\right) & =\left[\ldots[\left(\mathcal{H}^{-1}(\square)\right)^{m}, \underbrace{\left.\mathcal{H}^{-1}(\tau)\right], \ldots, \mathcal{H}^{-1}(\tau)}_{\text {j-times }}]\right. \\
& =i^{m+j}\left[\ldots[\check{\square}^{m}, \underbrace{, \check{\tau}}_{j \text {-times }}], \ldots, \check{\tau}\right]=i^{m+j} \nabla_{j}^{m} .
\end{aligned}
$$

Then, taking into account theorem 6 and theorem 11, the theorem follows.

Finally, taking into account that symmetries of $\mathcal{E}_{E D}$ are linear in $u_{\sigma}$ (see proposition 12), for each couple $\Delta, \nabla$ of recursion operators of $\mathcal{E}_{E D}$, we have that $[\Delta, \nabla](u)$ $=-\{\Delta(u), \nabla(u)\}$. Then, in view of previous theorem, we get the Lie structure of $\operatorname{NSym}\left(\mathcal{E}_{E D}\right)$.

\section{REFERENCES}

[1] G.W. Bluman and S. Kumei, Symmetries and differential equations, applied mathematical sciences 81 (Springer-Verlag, New York, 1989).

[2] A. V. Bocharov, V. N. Chetverikov, S. V. Duzhin, N. G. Khor'kova, I. S. Krasil'shchik, A. V. Samokhin, Yu. N. Torkhov, A. M. Verbovetsky and A. M. Vinogradov, Symmetries and conservation laws for differential equations of mathematical physics, (I. S. Krasilśhchik and A. M. Vinogradov, Editors) (Amer. Math. Soc., Providence, R.I., 1999).

[3] F. Bruhat and H. Whitney, 'Quelques propriétés fondamentales des ensembles analytiques-réels', Comment. Math. Helv. 33 (1959), 132-160.

[4] D. Catalano Ferraioli, G. Manno and F. Pugliese, 'Contact symmetries of the elliptic Euler-Darboux equation', Note Mat. 23 (2004), 3-14.

[5] J. Dieudonné, Foundations of modern analysis (Academic Press, New York and London, 1960).

[6] H. Grauert and K. Fritzsche, Several complex variables, Graduate Texts in Mathematics 38 (Springer-Verlag, New York, Heidelberg, 1976).

[7] I.S. Krasilshchik and A.M. Verbovetsky, 'Homological methods in equations of mathematical physics', (Open Education and Sciences, Opava (Czech Rep.)). math.DG/9808130.

[8] R.S. Kulkarni, 'On complexifications of differentiable manifolds', Invent. Math. 44 (1978), 46-64.

[9] P.J. Olver, Application of Lie groups to differential equations (Springer-Verlag, New York, 1993).

[10] C. Rogers and W. F. Shadwick, Baecklund transformations and their applications, Mathematics in Science and Engineering, 161 (Academic Press, New York, London, 1982).

[11] V. Rosenhaus, 'The unique determination of the equation by its invariance group and field-space symmetry', Algebras Groups Geom. 3 (1986), 148-166.

[12] D. J. Saunders, The geometry of jet bundles (Cambridge Univ. Press, Cambridge, 1989).

[13] V.E. Shemarulin, 'Higher symmetry algebra structure and local equivalences of Euler-Darboux equations', (Russian), Dokl. Akad. Nauk. 330 (1993), 24-27. Translation in Russian Acad. Sci. Dokl. Math. 47 (1993) 383-388. 
[14] V.E. Shemarulin, 'Higher symmetries and conservation laws of Euler-Darboux equations', in Geometry in partial differential equations (World Sci. Publishing, River Edge, NJ, 1994), pp. 389-422.

[15] V.E. Shemarulin, 'Higher symmetry algebra structures and local equivalences of Euler-Darboux equations', in The interplay between differential geometry and differential equations, Amer. Math. Soc. Transl. Ser. 2167 (Amer. Math. Soc., Providence, RI, 1995), pp. 217-243.

[16] G. Sparano, G. Vilasi and A.M. Vinogradov, 'Vacuum Einstein metrics with bidimensional Killing leaves I: local aspects', Diff. Geom. Appl. 16 (2002), 95-120.

[17] G. Sparano, G. Vilasi and A.M. Vinogradov, 'Vacuum Einstein metrics with bidimensional Killing leaves II: global aspects', Diff. Geom. Appl. 17 (2002), 15-35.

[18] A. Tognoli, 'Sulla classificazione dei fibrati analitici reali', Ann. Scuola Norm. Sup. Pisa 21 (1967), 709-743.

[19] G. Tomassini, 'Tracce delle funzioni olomorfe sulle sottovarietà analitiche reali d'una varietà complessa', Ann. Scuola Norm. Sup. Pisa 20 (1966), 31-43.

[20] A.M. Vinogradov, 'Local symmetries and conservation laws', Acta Appl. Math. 2 (1981), 21 78.

[21] A.M. Vinogradov, 'An informal introduction to the geometry of jet spaces', Rend. Sem. Fac. Sci. Univ. Cagliari 58 (1988), 301-333.

Dip. di Matematica

Università di Milano

via Saldini

20133 Milano

Italy

e-mail: catalano@mat.unimi.it
Dip. di Matematica

Università di Lecce

via per Arnesano

73100 Lecce

Italy

e-mail: gianni.manno@unile.it

Dip. di Matematica e Informatica

Università di Salerno

via Ponte don Melillo

84084 Fisciano (SA) Italy

e-mail: fpugliese@unisa.it 